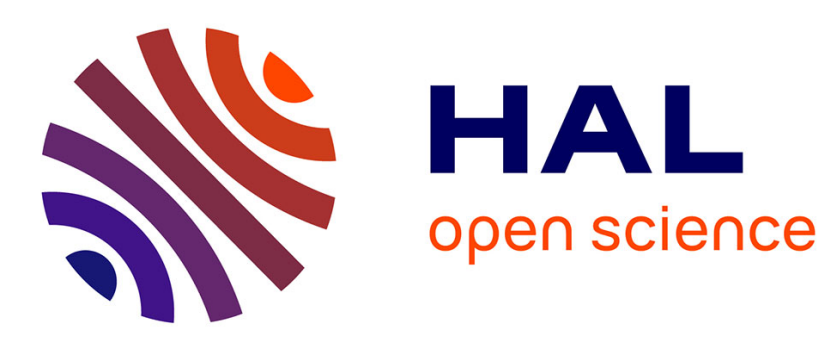

\title{
Line Profiles of the Calcium I Resonance Line in Cool Metal-polluted White Dwarfs
}

Simon Blouin, Nicole F. Allard, Thierry Leininger, Florent X. Gadéa, Patrick Dufour

\section{- To cite this version:}

Simon Blouin, Nicole F. Allard, Thierry Leininger, Florent X. Gadéa, Patrick Dufour. Line Profiles of the Calcium I Resonance Line in Cool Metal-polluted White Dwarfs. The Astrophysical Journal, 2019, 875 (2), pp.137. 10.3847/1538-4357/ab1266 . hal-02156152

\section{HAL Id: hal-02156152 https://hal.science/hal-02156152}

Submitted on 2 Sep 2019

HAL is a multi-disciplinary open access archive for the deposit and dissemination of scientific research documents, whether they are published or not. The documents may come from teaching and research institutions in France or abroad, or from public or private research centers.
L'archive ouverte pluridisciplinaire HAL, est destinée au dépôt et à la diffusion de documents scientifiques de niveau recherche, publiés ou non, émanant des établissements d'enseignement et de recherche français ou étrangers, des laboratoires publics ou privés. 


\title{
Line Profiles of the Calcium I Resonance Line in Cool Metal-polluted White Dwarfs
}

\author{
S. Blouin ${ }^{1}(1)$, N. F. Allard ${ }^{2,3}$, T. Leininger ${ }^{4}$, F. X. Gadéa ${ }^{4}$, and P. Dufour ${ }^{1}$ \\ ${ }^{1}$ Département de Physique, Université de Montréal, Montréal, QC H3C 3J7, Canada; sblouin@astro.umontreal.ca, dufourpa@astro.umontreal.ca \\ ${ }^{2}$ GEPI, Observatoire de Paris, Université PSL, CNRS, UMR 8111, 61 avenue de l'Observatoire, F-75014 Paris, France; nicole.allard@obspm.fr \\ ${ }^{3}$ Sorbonne Université, CNRS, UMR 7095, Institut d'Astrophysique de Paris, 98bis boulevard Arago, F-75014 Paris, France \\ ${ }^{4}$ Laboratoire de Physique et Chimie Quantique, UMR 5626, Université de Toulouse (UPS) and CNRS, 118 route de Narbonne, F-31400 Toulouse, France
}

\begin{abstract}
Metal-polluted white dwarfs (DZ stars) are characterized by a helium-rich atmosphere contaminated by heavy elements traces originating from accreted rocky planetesimals. As a detailed spectroscopic analysis of those objects can reveal the composition of the accreted debris, there is a great interest in developing accurate DZ atmosphere models. However, the coolest DZ white dwarfs are challenging to model due to the fluidlike density of their atmospheres. Under such extreme conditions, spectral absorption lines are heavily broadened by interactions with neutral helium, and it is no longer justified to use the conventional Lorentzian profiles. In this work, we determine the theoretical profiles of the Ca I resonance line (the most prominent spectral line for the coolest DZ white dwarfs) in the dense atmospheres of cool DZ white dwarfs. To do so, we use a unified theory of collisional line profiles and accurate ab initio potential energies and transition dipole moments for the CaHe molecule. We present the resulting profiles for the full range of temperatures and helium densities relevant for the modeling of cool, metal-polluted white dwarfs (from 3000 to $6000 \mathrm{~K}$ and from $10^{21}$ to $10^{23} \mathrm{~cm}^{-3}$ ). We also implement these new profiles in our atmosphere models and show that they lead to improved fits to the Ca I resonance line of the coolest DZ white dwarfs.
\end{abstract}

Key words: line: profiles - opacity - stars: individual (SDSS J080440.63+223948.6, WD J2356-209, WD 2251-070) - white dwarfs

\section{Introduction}

After exhausting their supplies of nuclear fuel, the vast majority of main-sequence stars will end their lives as white dwarfs. These stellar remnants are then condemned to a slow cooling that will extend over billions of years. White dwarfs are compact objects $\left(\approx 0.6 M_{\odot}\right.$ compressed into a volume similar to that of the Earth), and thus, they are characterized by a very intense surface gravity. Because of this strong gravitational field (typically, $\log g=8$ ), atomic species stratify according to their weights. Hence, only the lightest elements-hydrogen and helium-are found in the atmospheres of most white dwarfs.

A particularly interesting exception to this rule is the existence of DZ stars, which are white dwarfs whose heliumrich atmospheres are contaminated by traces of heavy elements (e.g., $\mathrm{Mg}, \mathrm{Ca}, \mathrm{Na}, \mathrm{Fe}$, and $\mathrm{Si}$ ) that are detected thanks to their absorption lines. The existence of those objects is a priori incompatible with the very efficient gravitational settling at work in white dwarfs. In particular, these heavy elements are expected to sink below the photosphere within timescales that are much smaller than the age of the white dwarf (Paquette et al. 1986; Koester 2009).

The accepted scenario for the existence of DZ white dwarfs is that heavy elements were recently (or are being) added to the atmospheres of those objects through the accretion of rocky planetesimals (e.g., see the reviews of Jura \& Young 2014; Farihi 2016). This scenario is now supported by the detection of infrared excesses that signal the presence of circumstellar debris disks around dozens of white dwarfs (Melis et al. 2010; Brinkworth et al. 2012; Rocchetto et al. 2015) and the discovery of planetary transits in the light curve of WD 1145 +017 (Vanderburg et al. 2015). Metal-polluted white dwarfs constitute a unique observational window into the bulk composition of planetesimals (comets, asteroids, and dwarf planets) outside our solar system. Using atmosphere models to fit the observed spectra of DZ white dwarfs, we can determine the chemical composition of their atmospheres and trace back the composition of the accreted planetesimals (Zuckerman et al. 2007; Farihi et al. 2013; Hollands et al. 2018).

For the coolest $\left(T_{\text {eff }} \approx 4000 \mathrm{~K}\right.$ ) - and thus, oldest-white dwarfs, very few metal spectral lines remain visible. In this regime, one of the most prominent lines is the $\mathrm{Ca}$ I resonance line at $4226 \AA$ (see, for instance, WD 2251-070 in Dufour et al. 2007). Properly modeling this absorption feature is of utmost importance if we want to precisely determine the composition of these old objects. For instance, one of the oldest DZ white dwarf known to date (WD J2356-209, with a cooling age of $\approx 8 \mathrm{Gyr}$ ) has been shown to have an abnormally high $\mathrm{Na} / \mathrm{Ca}$ abundance ratio, which raises a number of questions about the origin of the planetesimal that it accreted (Blouin et al. 2019). The Ca abundance in the atmosphere of this object was inferred from a fit to its $\mathrm{Ca}$ I resonance line. Therefore, the determination of the atmospheric composition of this white dwarf and any conclusions about the nature of the accreted planetesimal are strongly influenced by the quality of our Ca I $4226 \AA$ line profiles.

However, obtaining accurate absorption line profiles under the physical conditions found at the photosphere of those cool, helium-rich white dwarfs is challenging. Because of their very transparent atmospheres, cool DZ white dwarfs have photospheres where the density can reach $n_{\mathrm{He}}=10^{23} \mathrm{~cm}^{-3}$ (Blouin et al. 2018a). Under such fluidlike conditions, the wings of heavy-element spectral lines are strongly broadened by interactions with neutral helium, and Lorentzian profiles do not allow a satisfactory fit to the observed spectra (e.g., Allard et al. 2016). It is then necessary to implement a unified line shape theory (Allard et al. 1999) to obtain the right spectral line 
profiles. This approach requires prior knowledge, for each molecular state involved in the transition, of the potential energy and the variation of the dipole moment with respect to the atom-atom separation.

The accuracy of the line profiles is strongly affected by the quality of the atomic data from which they are computed. The study of SDSS J080440.63+223948.6 (SDSS J0804+2239) by Blouin et al. (2018b) offers a good example of the importance of using high-quality ab initio data for these calculations. As no accurate potential energies for the $\mathrm{CaHe}$ molecule were available, Blouin et al. (2018b) tried to model the Ca I resonance line of SDSS J0804+2239 using approximate potential energy curves (PECs). The limitations of their approximate potentials were quite obvious, as they were unable to obtain a completely satisfactory fit to this absorption line.

For their analysis of WD J2356-209, Blouin et al. (2019) relied on more accurate $\mathrm{Ca}$ I $4226 \AA$ line profiles-thanks to the improved PECs of Hernando et al. (2008; M. Barranco 2018, private communication) - and achieved an excellent fit to the Ca I resonance line of this object. Still, Blouin et al. (2019) did not have access to the transition dipole moments and to the long-range portion of the PECs, which are both needed to obtain the most accurate line profiles. In this paper, we use state-of-the-art ab initio data to fill these gaps and obtain more accurate $\mathrm{Ca}$ I resonance line profiles. The new ab initio data on which this work relies are described in Section 2. In Section 3, we present our new $\mathrm{Ca}$ I resonance line profiles. We apply these new profiles to three cool DZ white dwarfs in Section 4, and our conclusions are given in Section 5.

\section{Ab Initio Data}

Line profile intensities are functions of both excited and ground-state interactions. Therefore, a precise determination of the electronic energies and optical transition dipole moments is crucial to accurately compute the $\mathrm{Ca}$ I resonance line profiles for the whole wavelength range. To obtain the necessary atomic data, we performed ab initio calculations with the Molpro package (Werner et al. 2012). All singlet states up to the $\mathrm{Ca}^{1} \mathrm{~S}(4 \mathrm{~s} 5 \mathrm{~s})+\mathrm{He}\left(1 \mathrm{~s}^{2}\right)$ asymptote have been involved (i.e., four $\Sigma$ states, two $\Pi$ states, and one $\Delta$ state). A large-core pseudopotential was used for Ca (Czuchaj et al. 1991) together with the usual Core Polarisation Potential approach (Müller et al. 1984). For Ca, the associated 8s8p7d basis set of Czuchaj et al. (1991) has been complemented by $7 \mathrm{f}$ and $3 \mathrm{~g}$ Gaussian functions; while for He, the spdfg aug-v5z-cc basis set has been used (Woon \& Dunning 1994). State-averaged complete active space self-consistent field (CASSCF) calculations with four active electrons in 11 orbitals (corresponding to the $4 \mathrm{~s}, 4 \mathrm{p}, 3 \mathrm{~d}$, and $5 \mathrm{~s}$ orbitals for $\mathrm{Ca}$ and the $1 \mathrm{~s}$ orbital for $\mathrm{He}$ ) were performed, followed by multireference configuration interaction (MRCI) calculations for each symmetry. The transition dipole moments were then computed using the averaged natural orbitals from the bra wavefunction.

The resulting potentials are reported in Figure 1. The ground state $\left(\mathrm{X}{ }^{1}{ }^{1} \Sigma\right)$ is mainly repulsive with a shallow van der Waals well, the $2{ }^{1} \Sigma$ and $2{ }^{1} \Pi$ (A) potentials are more attractive and similar to the ground-state potential of the $\mathrm{Ca}^{+} \mathrm{He}$ ion, and the $3^{1} \Sigma$ (B) potential is very repulsive. From the generalized Fermi model (Dickinson \& Gadéa 2002), because the electron $-\mathrm{He}$ scattering length is positive, repulsive effects are expected. Moreover, since the ${ }^{1} \mathrm{D}(4 \mathrm{~s} 3 \mathrm{~d})$ and ${ }^{1} \mathrm{P}(4 \mathrm{~s} 4 \mathrm{p})$



Figure 1. PECs for the $4 \mathrm{~s}$ and $4 \mathrm{p}$ states of the CaHe molecule. For comparison, the potentials computed by Hinde (2003) and Hernando et al. (2008) (which were used in Blouin et al. 2019) are also shown.

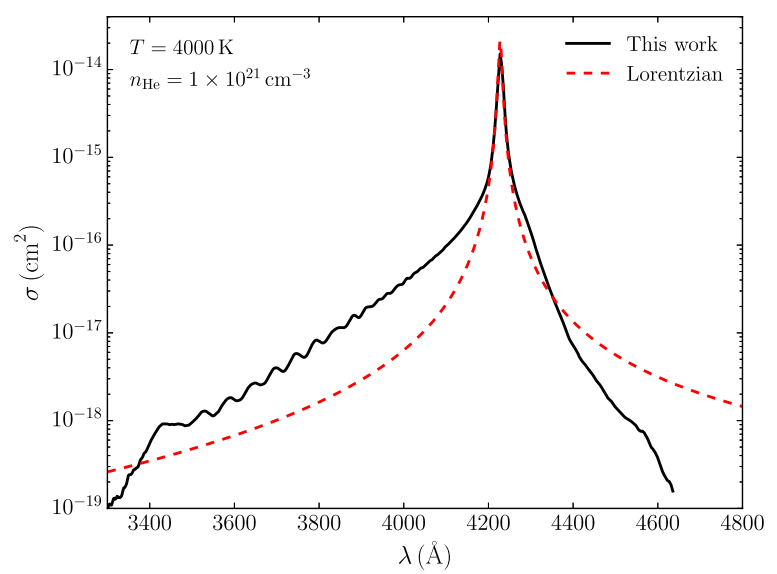

Figure 2. Comparison of the unified profile (solid black line) with the corresponding Lorentzian profile (dashed red line).

asymptotes are rather close in energy, it is not surprising that the highest $\mathrm{B}$ state $\left(3^{1} \Sigma\right)$ cumulates the repulsive interactions with $\mathrm{He}$, while the lower one does not and looks like the ground-state potential of the $\mathrm{Ca}^{+} \mathrm{He}$ ion.

\section{Line Profiles}

To compute the pressure broadening of the Ca I resonance line, we rely on the classical path expression derived by Allard et al. (1999). In contrast with other more usual approximations (Anderson 1952; Baranger 1958a, 1958b), the approach of Allard et al. takes the dependence of the electric dipole moment on the position of perturbers into account, which can significantly affect the spectral line shape (Allard et al. 1998a, 1998b).

\subsection{Low Densities}

Under sufficiently low perturber densities, the center of the spectral line is expected to be symmetric and described by a Lorentzian profile. This profile is defined by two parameters, a width and a shift, which have a linear dependence on the density. We find that the core of the line is adequately described by a Lorentzian up to $n_{\mathrm{He}}=10^{21} \mathrm{~cm}^{-3}$ (Figure 2). Using the impact limit of the general calculation of the autocorrelation function (Allard et al. 1999), we computed the 


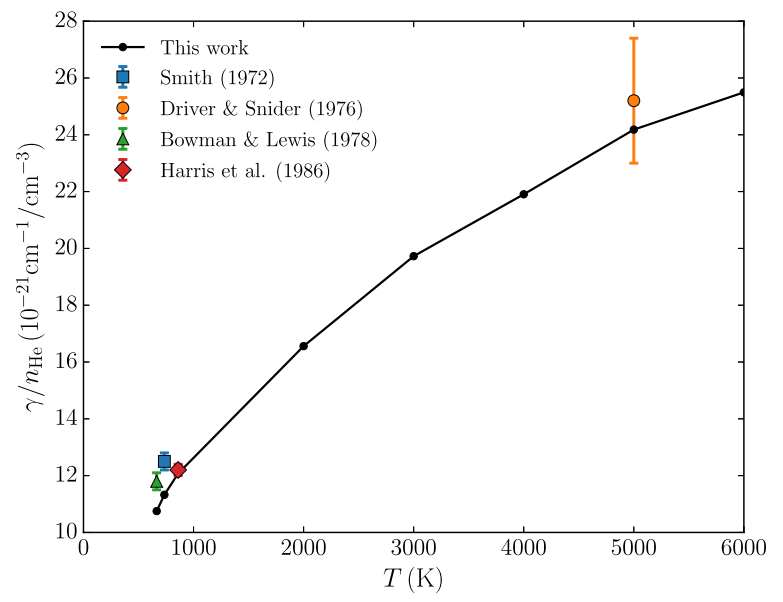

Figure 3. Variation with temperature of the HWHM of the Ca I resonance line perturbed by He collisions. Our results are shown in black, and the error bars represent experimental measurements.

line parameters that characterize this Lorentzian core. As shown in Figure 3, the resulting theoretical broadening parameters are in reasonable agreement with laboratory measurements (Smith 1972; Driver \& Snider 1976; Bowman \& Lewis 1978; Harris et al. 1986). Note that the error bars on the experimental data are probably underestimated, since the three points at low temperatures appear to imply an improbable temperature dependence.

While the core of the resonance line is adequately described by a Lorentzian profile at $n_{\mathrm{He}}=10^{21} \mathrm{~cm}^{-3}$, it is of course not the case for the wings (Figure 2). The $\mathrm{X}-\mathrm{B}$ transition is responsible for the shape of the blue wing, and the $\mathrm{X}-\mathrm{A}$ transition affects the red side. The small maximum in the blue wing near $3400 \AA$ is a direct consequence of the shoulder of the B state PEC at $R=2.2 \AA$, which gives rise to a local maximum of $\Delta V(R)$ at $\Delta V=5800 \mathrm{~cm}^{-1}$. As satellite lines occur at frequencies corresponding to extrema of $\Delta V$, a satellite feature appears at $\Delta \omega=5800 \mathrm{~cm}^{-1}$ (which corresponds to $\lambda \simeq 3400 \AA$ ).

\subsection{High Densities}

The coolest DZ white dwarfs are characterized by photospheric densities that can go up to $n_{\mathrm{He}}=10^{23} \mathrm{~cm}^{-3}$, with much of their line-forming regions located at densities exceeding $10^{21} \mathrm{~cm}^{-3}$ (Figure 4). As those cool objects are precisely the ones that show a prominent $\mathrm{Ca}$ I resonance line, there is a strong astrophysical interest in properly modeling its line shape under such high-density conditions.

Given the high perturber densities involved, simultaneous collisions are very frequent. This implies that many-body collisions must be included in our calculation of the Ca I resonance line pressure broadening, and this is why we rely on the autocorrelation formalism of Allard et al. (1999). This approach has already proved successful for the modeling of spectral lines in cool DZ white dwarfs (e.g., Allard et al. 2016, 2018). Another proof of the accuracy of the Allard et al. (1999) formalism has been provided by the study of He doped with alkali atoms, which has recently been the subject of active study (e.g., Hernando et al. 2010; Mateo et al. 2011). In such experiments, large He clusters produced in a supersonic jet are doped with alkali atoms and are characterized by laser-induced fluorescence. In the case of $\mathrm{Na}$ atoms attached to He droplets

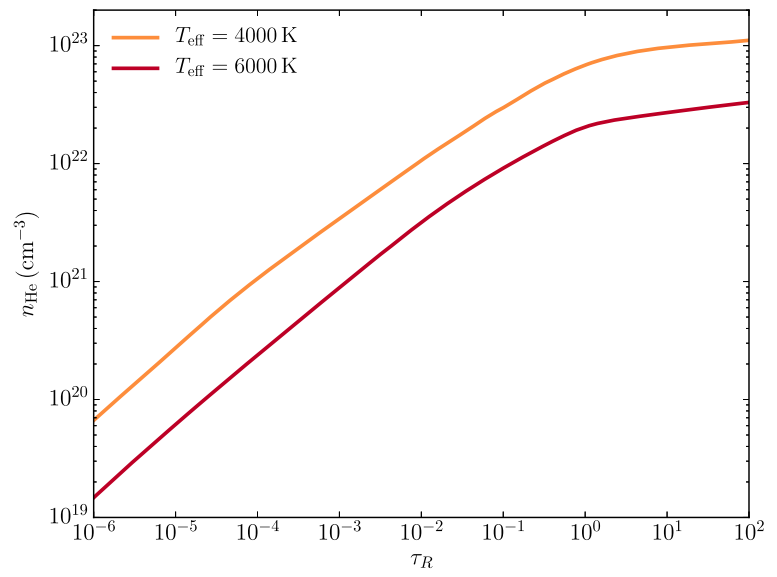

Figure 4. He density as a function of the Rosseland mean optical depth for two models of cool DZ atmospheres. Note that a hydrogen-free atmosphere with a calcium abundance of $\log \mathrm{Ca} / \mathrm{He}=-10$ and a surface gravity of $\log g=8$ was assumed for both models. The abundance ratios of all other heavy elements were scaled to the abundance of $\mathrm{Ca}$ to match the abundance ratios measured in CI chondrites (Lodders 2003).

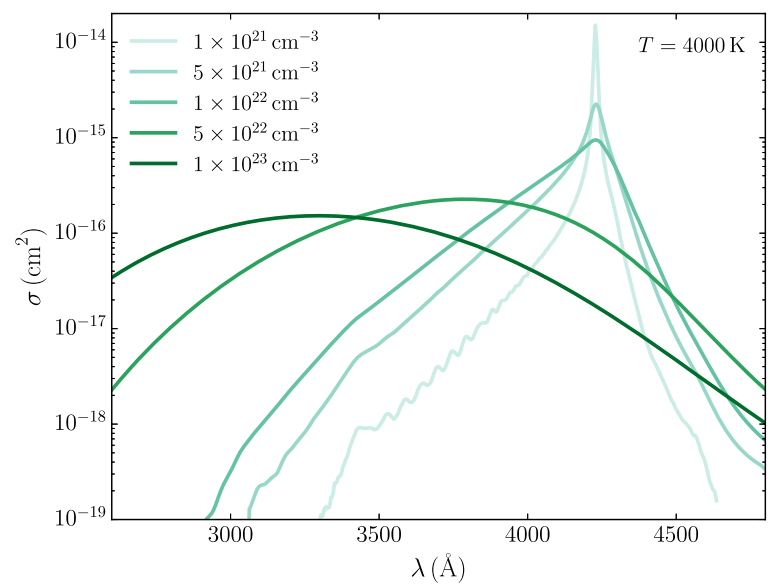

Figure 5. Density dependence of the Ca I resonance line profiles. A constant temperature of $T=4000 \mathrm{~K}$ is assumed.

(Stienkemeier et al. 1996), Allard et al. (2013) have shown that calculations performed within the autocorrelation framework can successfully explain the laboratory spectra. Moreover, the absorption spectra obtained by this approach was shown to be consistent with those obtained from path integral Monte Carlo simulations and the Franck-Condon approximation (Nakayama \& Yamashita 2001).

Figure 5 shows the results of our calculations for the evolution of the $\mathrm{Ca}$ I resonance line profile with increasing $\mathrm{He}$ density. Clearly, above $10^{21} \mathrm{~cm}^{-3}$, the profiles become strongly asymmetric and are shifted toward smaller wavelengths. Note that, as expected, the maximum of the line shifts toward the satellite line at $3400 \AA$. Apart from being strongly affected by the perturber density, line profiles can also be affected by the temperature of the medium (Allard et al. 2004). However, for the temperature range relevant to cool DZ atmospheres, we find that the profiles are not very sensitive to the temperature (Figure 6). 


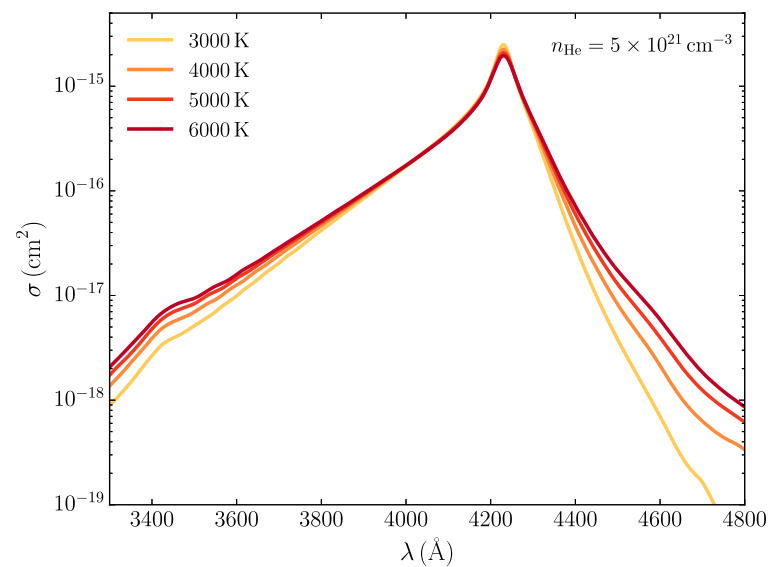

Figure 6. Temperature dependence of the $\mathrm{Ca}$ I resonance line profiles. A constant density of $n_{\mathrm{He}}=5 \times 10^{21} \mathrm{~cm}^{-3}$ is assumed.

\section{Astrophysical Applications}

\subsection{SDSS J0804+2239}

A comprehensive analysis of the DZ white dwarf SDSS J0804+2239 was recently presented in Blouin et al. (2018b). Thanks to the improved constitutive physics of their atmosphere models, they were able to find a satisfactory fit to both its metal spectral lines and its spectral energy distribution, which is affected by $\mathrm{H}_{2}-\mathrm{He}$ collision-induced absorption. They found an effective temperature of $T_{\text {eff }}=4970 \pm 100 \mathrm{~K}$, a surface gravity of $\log g=7.98 \pm 0.05$, and number abundances of $\log \mathrm{H} / \mathrm{He}=-1.6 \pm 0.2 \quad$ and $\log \mathrm{Ca} / \mathrm{He}=-10.0 \pm 0.1$. However, their spectroscopic fit of SDSS J0804+2239 was tarnished by their inability to adequately fit the blue wing of the $\mathrm{Ca}$ I resonance line, which they attributed to the poor quality of the CaHe PECs used to compute the profiles of this spectral line. Using the same atmospheric parameters ( $T_{\text {eff }}, \log g$, and individual abundances) as those found by Blouin et al. (2018b), we fitted the Ca lines of SDSS J0804+2239 with our improved Ca I $4226 \AA$ profiles. As shown in Figure 7, these new profiles allow us to obtain an improved spectroscopic fit to the blue wing of the $\mathrm{Ca}$ I resonance, solving the problem identified by Blouin et al. (2018b).

\subsection{WD J2356-209}

WD J2356-209 is a very cool DZ white dwarf whose visible spectrum is dominated by a very broad sodium feature (Salim et al. 2004). Blouin et al. (2019) have obtained a fit that is in very good agreement with observations across all wavelengths, and they have shown that WD J2356-209 has a record $\mathrm{Na} / \mathrm{Ca}$ abundance ratio. However, some uncertainty persisted as the $\mathrm{CaI}$ resonance profiles were computed assuming constant transition dipole moments and as the longrange portion of the CaHe PECs was not available. Thanks to our new ab initio data on the CaHe molecule, the uncertainties that could potentially plague the $\mathrm{Ca}$ abundance determination are no longer present. Using our Ca I $4226 \AA$ profiles, we performed a new spectroscopic fit of WD J2356-209 (Figure 8), and we found virtually the same abundances. In particular, we found a calcium abundance of $\log \mathrm{Ca} / \mathrm{He}=-9.4 \pm 0.1$, which is within the uncertainties of the $-9.3 \pm 0.1$ value of Blouin et al. (2019). Therefore, their conclusions are unchanged despite the addition of our new

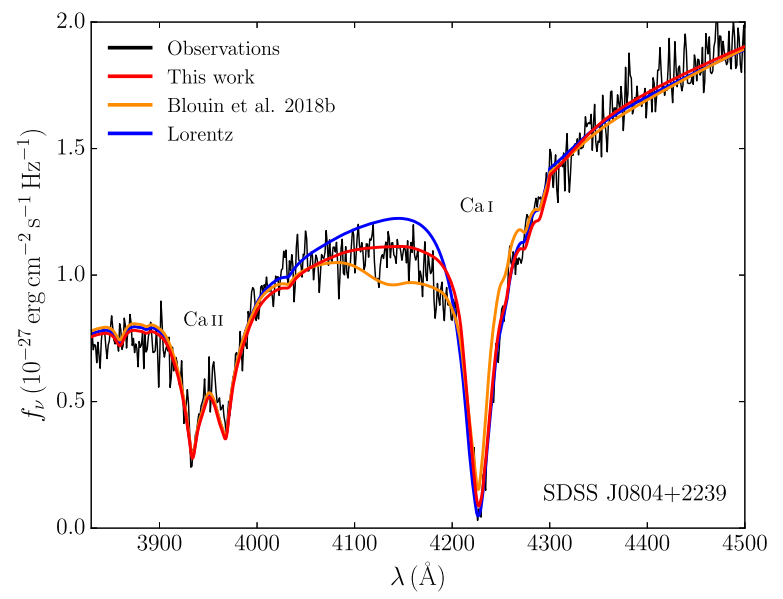

Figure 7. Comparison of synthetic spectra computed with different line profiles for the Ca I $4226 \AA$ transition. Observations of J0804+2239 are in black, results found when assuming a Lorentzian profile are in blue, and synthetic spectra obtained with the unified line shape theory of Allard et al. (1999) are in orange for the case where approximate $\mathrm{CaHe}$ potentials were used (Blouin et al. $2018 \mathrm{~b}$ ) and in red for the case where the high-quality $\mathrm{CaHe}$ potentials presented in this work were used. All synthetic spectra were computed assuming the atmospheric parameters of SDSS J0804+2239 found in Blouin et al. (2018b; i.e., $T_{\text {eff }}=4970 \mathrm{~K}, \log g=7.98, \log \mathrm{H} / \mathrm{He}=-1.6$, and $\log \mathrm{Ca} / \mathrm{He}=-10.0$ ). Note that in all cases, the $\mathrm{Ca} \mathrm{II} \mathrm{H}$ and $\mathrm{K}$ lines are computed with the profiles described in Allard \& Alekseev (2014).

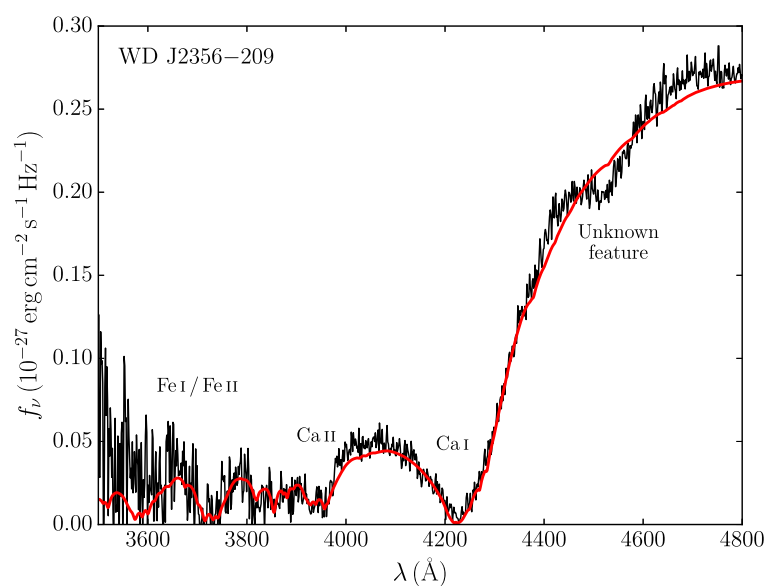

Figure 8. Spectroscopic fit of the Ca I resonance line of WD J2356-209. As in Blouin et al. (2019), $T_{\text {eff }}=4040 \mathrm{~K}, \log g=7.98$, and $\log \mathrm{Na} / \mathrm{He}=-8.3$. However, the calcium abundance is now $\log \mathrm{Ca} / \mathrm{He}=-9.4 \pm 0.1$, which is slightly lower than the value reported by Blouin et al. (2019).

profiles: WD J2356-209 does have a uniquely high $\mathrm{Na} / \mathrm{Ca}$ abundance ratio. We note, however, that our new profiles cannot account for the absorption feature near $4500 \AA$. The nature of this absorption feature remains unknown (see Section 4.3 of Blouin et al. 2019 for a comprehensive discussion on this issue).

\subsection{WD $2251-070$}

After WD J2356-209, WD 2251-070 is the second coolest known DZ white dwarf. Its spectrum is quite different from that of WD J2356-209, as it has a prominent Ca I resonance line but does not show a strong Na D doublet (Blouin et al. 2019, Figure 5). While reasonable fits to its spectrum have been obtained in previous studies (Kapranidis \& Liebert 1986; Dufour et al. 2007), atmosphere model codes used to analyze 
this object relied on constitutive physics that are not totally appropriate for the dense atmosphere of this cool white dwarf.

In particular, the analysis of Kapranidis \& Liebert (1986) relied on models that assumed a Thomas-Fermi equation of state (Kapranidis 1983). Because of this assumption, they found that electron thermal conduction was an important energy transport mechanism, a conclusion that does not hold if we use more realistic equations of state (Bergeron et al. 1995; Kowalski et al. 2007). Also, Kapranidis \& Liebert (1986) computed their synthetic spectra from pure helium atmosphere structures, thus ignoring the impact of heavy elements on the pressure and temperature stratification. Moreover, a simple Lorentzian profile was assumed for the $\mathrm{Ca}$ I resonance line. Despite all those simplifying assumptions, they managed to obtain an excellent fit to the resonance line (with $T_{\text {eff }}=4500 \mathrm{~K}$ and $\log \mathrm{Ca} / \mathrm{He}=-6.3)$ and were even able to find a good agreement to the unknown feature at $4500 \AA$. Unfortunately, no detail regarding this puzzling structure was given, so we cannot know how they were able to explain it.

Dufour et al. (2007) revisited WD 2251-070 using more realistic atmosphere models, where heavy elements are included in the calculation of the atmosphere structures. That being said, a number of unjustified approximations remained, such as the ideal gas law, the ideal Saha ionization equilibrium, and Lorentzian profiles. Their best solution $\left(T_{\text {eff }}=4000 \mathrm{~K}\right.$ and $\log \mathrm{Ca} / \mathrm{He}=-10.5$ ) was in good agreement with the photometry and with the $\mathrm{Ca}$ I resonance line (but they were unable to explain the absorption feature near $4500 \AA$ ).

Here, we revisit this star using our improved models (Blouin et al. 2018a) and our new Ca I resonance line profiles. To do so, we use the spectroscopic observations of Dufour et al. (2007), the Gaia data release 2 (DR2) parallax measurement $(\pi=117.15 \pm 0.05$ mas; Gaia Collaboration 2016, 2018), photometry from Bergeron et al. (1997), the Panoramic Survey Telescope And Rapid Response System, Chambers et al. (2016), and the Two Micron All-Sky Survey. Our best fit to the spectroscopic and photometric observations is shown in Figure 9, and the corresponding atmospheric parameters are given in Table 1.

To obtain this solution, we used the photometric technique (Bergeron et al. 1997) to determine $T_{\text {eff }}$ and $\log g$. More precisely, the solid angle $\pi(R / D)^{2}$ and the effective temperature were found by adjusting the model fluxes to the photometric observations. From the solid angle, we computed the white dwarf radius $R$ ( $D$ is given by the Gaia parallax) and, from there, we found the mass and the surface gravity using the evolutionary models of Fontaine et al. (2001). Using the parameters given by the photometric fit, we then adjusted the $\mathrm{Ca}$ abundance to the spectroscopy. As the abundance obtained from this spectroscopic fit was different from that originally assumed, we repeated the whole fitting procedure (the photometric and the spectroscopic fits) until we reached a consistent solution. Additionally, as no collision-induced absorption is visible in the infrared photometry, we were able to constrain the hydrogen abundance to $\log \mathrm{H} / \mathrm{He}<-4.5$.

As shown in Figure 9, we are able to find a good fit to most of the spectrum of WD 2251-070 (with the notable exception of the unknown absorption feature near $4500 \AA$ ). In particular, our best solution is in good agreement with the observations in the region located between the $\mathrm{Ca}$ II $\mathrm{H} \& \mathrm{~K}$ doublet and the $\mathrm{Ca}$ I resonance line, whereas the best solution of Dufour et al. (2007) was significantly above the observed spectrum in this

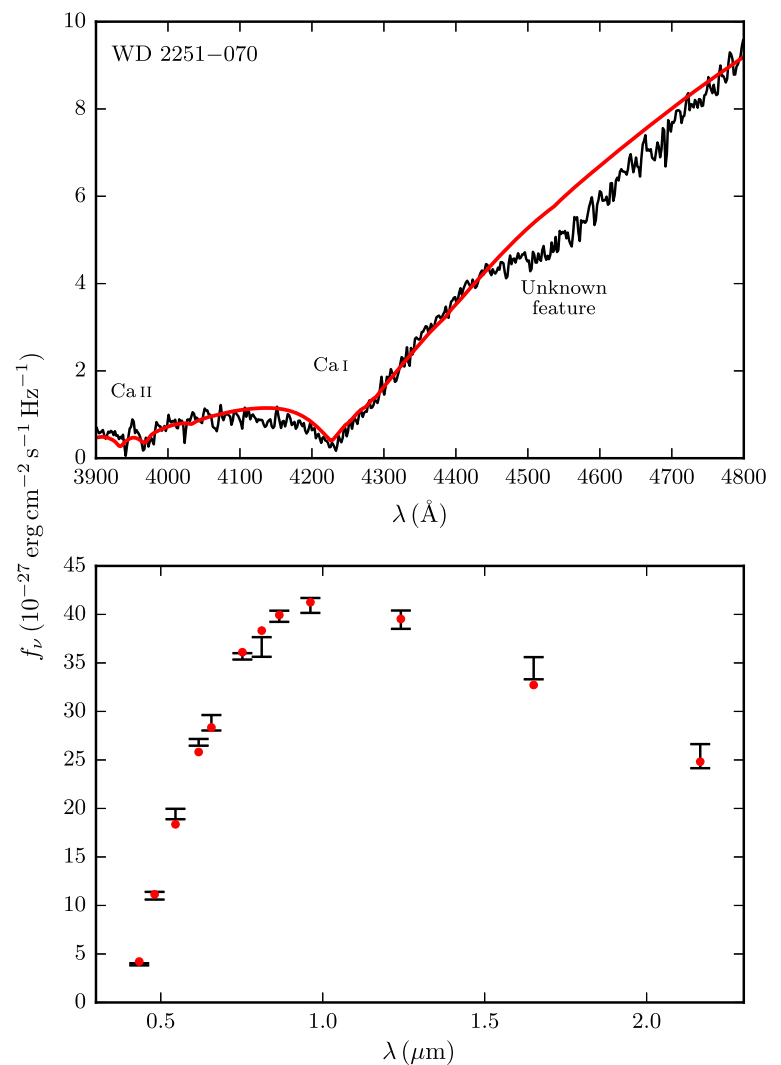

Figure 9. Our best solution for WD 2251-070. The top panel shows our spectroscopic fit, and the bottom panel shows our fit to the BVRI, grizy, and $J H K$ photometry.

Table 1

WD 2251-070 Atmospheric Parameters

\begin{tabular}{lc}
\hline \hline Parameter & Value \\
\hline$T_{\text {eff }}$ & $4170 \pm 90 \mathrm{~K}$ \\
$\log g$ & $8.06 \pm 0.08$ \\
$\log \mathrm{H} / \mathrm{He}$ & $<-4.5$ \\
$\log \mathrm{Ca} / \mathrm{He}$ & $-9.8 \pm 0.2$ \\
\hline
\end{tabular}

region. Moreover, unlike Kapranidis \& Liebert (1986), we find a reasonable match to the weak $\mathrm{Ca}$ II $\mathrm{H} \& \mathrm{~K}$ doublet.

\section{Conclusion}

$\mathrm{Ca}$ I resonance line profiles suitable for the extreme densities of the atmospheres of cool, metal-polluted white dwarfs were presented. These new profiles were computed using state-ofthe-art $a b$ initio data and a unified theory of collisional line profiles. At low densities, they are compatible with laboratory measurements and, at high densities, they are heavily broadened and shifted toward lower wavelengths. We implemented these new profiles in our model atmosphere code and showed how they lead to good spectroscopic fits for three cool DZ white dwarfs (SDSS J0804+2239, WD J2356-209 and WD 2251-070). However, in the case of WD J2356-209 and WD 2251-070, there remains a discrepancy between our models and the observations, as there seems to be missing an absorption source in our models near $4500 \AA$. 
The CaHe potentials and dipole moments can be obtained upon request from $\mathrm{T}$. Leininger and the $\mathrm{Ca} \mathrm{I}$ resonance line profiles from N. F. Allard.

This work was supported in part by NSERC (Canada) and the Fund FRQNT (Québec).

This work has made use of data from the European Space Agency (ESA) mission Gaia (https://www.cosmos.esa.int/ gaia), processed by the Gaia Data Processing and Analysis Consortium (DPAC, https://www.cosmos.esa.int/web/gaia/ dpac/consortium). Funding for the DPAC has been provided by national institutions, in particular the institutions participating in the Gaia Multilateral Agreement.

\section{ORCID iDs}

S. Blouin (iD https://orcid.org/0000-0002-9632-1436

\section{References}

Allard, N. F., \& Alekseev, V. A. 2014, AdSpR, 54, 1248

Allard, N. F., Drira, I., Gerbaldi, M., Kielkopf, J., \& Spielfiedel, A. 1998a, A\&A, 335, 1124

Allard, N. F., Kielkopf, J., \& Feautrier, N. 1998b, A\&A, 330, 782

Allard, N. F., Kielkopf, J. F., Blouin, S., et al. 2018, A\&A, 619, A152

Allard, N. F., Kielkopf, J. F., \& Loeillet, B. 2004, A\&A, 424, 347

Allard, N. F., Leininger, T., Gadéa, F. X., Brousseau-Couture, V., \& Dufour, P. 2016, A\&A, 588, A142

Allard, N. F., Nakayama, A., Spiegelman, F., Kielkopf, J. F., \& Stienkemeier, F. 2013, EPJD, 67, 52

Allard, N. F., Royer, A., Kielkopf, J. F., \& Feautrier, N. 1999, PhRvA, 60, 1021

Anderson, P. W. 1952, PhRv, 86, 809

Baranger, M. 1958a, PhRv, 111, 481

Baranger, M. 1958b, PhRv, 111, 494

Bergeron, P., Ruiz, M. T., \& Leggett, S. K. 1997, ApJS, 108, 339

Bergeron, P., Saumon, D., \& Wesemael, F. 1995, ApJ, 443, 764

Blouin, S., Dufour, P., \& Allard, N. F. 2018a, ApJ, 863, 184

Blouin, S., Dufour, P., Allard, N. F., et al. 2019, ApJ, 872, 188

Blouin, S., Dufour, P., Allard, N. F., \& Kilic, M. 2018b, ApJ, 867, 16
Bowman, N. J., \& Lewis, E. L. 1978, JPhB, 11, 1703

Brinkworth, C. S., Gänsicke, B. T., Girven, J. M., et al. 2012, ApJ, 750, 86

Chambers, K. C., Magnier, E. A., Metcalfe, N., et al. 2016, arXiv:1612.05560

Czuchaj, E., Rebentrost, F., Stoll, H., \& Preuss, H. 1991, CPL, 182, 191

Dickinson, A. S., \& Gadéa, F. X. 2002, PhRvA, 65, 052506

Driver, R. D., \& Snider, J. L. 1976, ApJ, 208, 518

Dufour, P., Bergeron, P., Liebert, J., et al. 2007, ApJ, 663, 1291

Farihi, J. 2016, NewAR, 71, 9

Farihi, J., Gänsicke, B. T., \& Koester, D. 2013, Sci, 342, 218

Fontaine, G., Brassard, P., \& Bergeron, P. 2001, PASP, 113, 409

Gaia Collaboration 2016, A\&A, 595, A1

Gaia Collaboration 2018, A\&A, 616, A1

Harris, M., Lewis, E. L., McHugh, D., \& Shannon, I. 1986, JPhB, 19, 3207

Hernando, A., Barranco, M., Mayol, R., et al. 2010, JLTP, 158, 105

Hernando, A., Barranco, M., Mayol, R., Pi, M., \& Krośnicki, M. 2008, PhRvB, 77, 024513

Hinde, R. J. 2003, JPhB, 36, 3119

Hollands, M. A., Gänsicke, B. T., \& Koester, D. 2018, MNRAS, 477, 93

Jura, M., \& Young, E. D. 2014, AREPS, 42, 45

Kapranidis, S. 1983, ApJ, 275, 342

Kapranidis, S., \& Liebert, J. 1986, ApJ, 305, 863

Koester, D. 2009, A\&A, 498, 517

Kowalski, P. M., Mazevet, S., Saumon, D., \& Challacombe, M. 2007, PhRvB, 76, 075112

Lodders, K. 2003, ApJ, 591, 1220

Mateo, D., Hernando, A., Barranco, M., Mayol, R., \& Pi, M. 2011, PhRvB, 83, 174505

Melis, C., Jura, M., Albert, L., Klein, B., \& Zuckerman, B. 2010, ApJ, 722,1078

Müller, W., Flesch, J., \& Meyer, W. 1984, JChPh, 80, 3297

Nakayama, A., \& Yamashita, K. 2001, JChPh, 114, 780

Paquette, C., Pelletier, C., Fontaine, G., \& Michaud, G. 1986, ApJS, 61, 177

Rocchetto, M., Farihi, J., Gänsicke, B. T., \& Bergfors, C. 2015, MNRAS, 449, 574

Salim, S., Rich, R. M., Hansen, B. M., et al. 2004, ApJ, 601, 1075

Smith, G. 1972, JPhB, 5, 2310

Stienkemeier, F., Higgins, J., Callegari, C., et al. 1996, ZPhyD, 38, 253

Vanderburg, A., Johnson, J. A., Rappaport, S., et al. 2015, Natur, 526, 546

Werner, H.-J., Knowles, P. J., Knizia, G., Manby, F. R., \& Schütz, M. 2012 Wiley Interdisciplinary Reviews: Computational Molecular Science, 2, 242

Woon, D. E., \& Dunning, T. H., Jr. 1994, JChPh, 100, 2975

Zuckerman, B., Koester, D., Melis, C., Hansen, B. M., \& Jura, M. 2007, ApJ, 671,872 